\title{
Desnutrición y su relación con parasitismo intestinal en niños de una población de la Amazonia colombiana
}

\author{
Leonardo Elías Ordóñez, Esther Sofia Angulo \\ Hospital Sagrado Corazón de Jesús, La Hormiga, Putumayo, Colombia.
}

En el mundo y principalmente en los países en vía de desarrollo, los parásitos intestinales y la desnutrición causan gran morbilidad, especialmente entre la población infantil. El presente estudio se desarrolló en un poblado de la Amazonia colombiana con el fin de determinar la frecuencia de parásitos intestinales, de desnutrición y para establecer, si se encontraban, relaciones entre ellos. Fueron incluidos 237 niños de quienes se recolectaron cuatro muestras de materia fecal para análisis parasitológicos; se calcularon los índices antropométricos talla para la edad (HAZ), peso para la edad (WAZ) y peso para la talla (WHZ) con respecto de la población de referencia internacional recomendada por la Organización Mundial de la Salud y, adicionalmente, se aplicó una encuesta socioeconómica diseñada por el estado colombiano. Los resultados arrojaron que el $86,1 \%$ se encontraba parasitado y el $54,4 \%$ tenía dos o más parásitos. El 29,5\% del grupo presentó baja talla para la edad; $10,1 \%$ bajo peso para la edad y $2,5 \%$ bajo peso para la talla. HAZ y WAZ presentaron relación inversa con la edad y el número de parásitos, en tanto que el puntaje en la encuesta socioeconómica tuvo una relación directa con el número de parásitos e inversa con el índice HAZ; todas estas relaciones fueron estadísticamente significativas $(p<0,05)$. En conclusión, las frecuencias de desnutrición y parasitismo intestinal en esta población son superiores a las informadas para el resto del país. Al igual que en estudios previos, la edad, el parasitismo intestinal y el estado socioeconómico son variables que se relacionan significativamente con el estado nutricional.

Palabras clave: desnutrición, baja talla para la edad, bajo peso para la talla, parasitismo intestinal, poliparasitismo intestinal, antropometría.

\section{Malnutrition and its association with intestinal parasitism among children from a village in the Colombian Amazon region}

Intestinal parasites and malnutrition cause high morbidity among children in developing countries. A study to establish the associations between intestinal parasitism and undernourishment was undertaken in a village in the Colombian Amazon region. Two hundred thirty seven children each provided four stool samples for parasitic analysis. Anthropometric indices were calculated based on height and weight (HAZ), weight and age (WAZ) and height and weight (WHZ) using the international growth standards recommended by the World Health Organization. Of the 237 children, $86.1 \%$ were carrying parasites, $54 \%$ had 2 or more parasites, $30 \%$ were stunted, $10 \%$ were underweight, and $2.5 \%$ were wasting. HAZ and WAZ indices were inversely related to age and number of parasites. Socioeconomic status was positively correlated with the number of parasites and inversely related to the HAZ index $(p<0.05)$. In conclusion, frequencies of undernourishment and intestinal parasites among the sample population were higher than the national average for Colombia. As previous studies have indicated, age, intestinal parasitism and socio-economic level are variables significantly related to nutritional state.

Key words: undernourishment, stunting, wasting, intestinal parasitism, intestinal polyparasitism, anthropometry. 
La asociación entre desnutrición y los diferentes parásitos intestinales, así como los mecanismos de dicha asociación han sido difíciles de delinear. Son varios los artículos publicados que apoyan o refutan diferentes relaciones (1-11). Esto se debe a que el peso, la talla y, en general, el estado nutricional de un individuo a lo largo de su vida depende de la compleja interacción de una serie de variables entre las cuales están su propia información genética, patologías que pueden afectar su desarrollo desde la vida intrauterina, el nivel socioeconómico y las conductas alimentarias de la familia y la sociedad a las que pertenece, así como las patologías que retrasan su crecimiento y desarrollo durante la infancia, la niñez y la pubertad, entre las cuales el parasitismo intestinal es tan sólo una $(1,12)$. Sin embargo, a partir de estudios en modelos animales y estudios transversales y de intervención en humanos, se han establecido algunos mecanismos por los cuales los parásitos intestinales inducen o agravan la malnutrición. Estos incluyen, cada uno por separado o en combinación, los siguientes: disminución en la ingestión de alimentos (hiporexia/anorexia), mala digestión, mala absorción, pérdidas crónicas de nutrientes por heces (enteropatía perdedora de proteínas, pérdidas sanguíneas) y disminución en las reservas de hierro y otros micronutrientes $(1,2,7,10,11)$.

Algunos ensayos terapéuticos también apoyan dicha relación; el tratamiento antihelmíntico de niños en Kenya produjo mejora en su estado nutricional (determinado por antropometría) y en la capacidad física (medido mediante el Harvard Step Test) $(3,4)$. Sin embargo, el primer efecto mencionado no se observó en un estudio recientemente realizado en Bangladesh en niños con bajo nivel de parasitismo, básicamente por Ascaris lumbricoides y Trichuris trichiura (5). Adicionalmente, los estudios en Nicaragua y Jamaica muestran una relación entre parasitismo intestinal, malnutrición y retraso en el desarrollo psicomotor (evaluado mediante la escala Denver

\footnotetext{
Correspondencia:

Esther S. Angulo Martínez. Av 7 \#151-67 Apto 101. BogotáColombia. Teléfono: 6254356.

esther_sofiaa@hotmail.com

Recibido:06/08/02; aceptado: 05/11/02
}

II) (13), y mejoría en el desempeño escolar luego de la desparasitación antihelmíntica (14), lo cual sugiere que las infecciones por helmintos se asocian con déficit en el desarrollo en otras áreas, además de la del crecimiento físico.

Para el año 2000, la prevalencia estimada de baja talla para la edad era del $32,5 \%$; de bajo peso para la talla, $9 \%$, y de bajo peso para la edad, $27 \%$ entre los niños menores de 5 años de los países en vía de desarrollo, excluyendo China (15). Para ese mismo año, en Suramérica, el 12,6\% de los niños menores de 5 años presentaban baja talla para la edad (15). De manera similar, los helmintos intestinales constituyen un problema de salud pública a nivel mundial; para 1999, se estimaba que 1.472 millones de personas en el mundo estaban parasitadas por A. lumbricoides, 1.298 millones por uncinarias, 1.049 millones por T. trichiura, 70 millones por Strongyloides stercoralis, 77 millones por Taenia saginata y 10 millones por Taenia solium (16).

En Colombia, el estado nutricional de los niños menores de 5 años muestra una progresiva mejoría para todos los índices antropométricos en las últimas décadas, según los estudios efectuados por el Ministerio de Salud de Colombia entre 1965-1966, 1977-1980 y 1986-1989 y la encuesta nacional de demografía y salud realizada por Profamilia en 2000 (ENDS, 2000); dicho progreso en el estado nutricional podría ser el efecto de un impacto positivo de las estrategias socioeconómicas, de salud y de nutrición desarrolladas en los últimos años en el país $(17,18)$; además, este cambio ha ido paralelo a la mejoría en otros indicadores sociales y económicos observada entre 1965 y 1989 (17). Sin embargo, hay que tener cautela al interpretar los resultados del último estudio como representativos del estado nutricional actual de los niños del país, ya que en la muestra de la ENDS 2000 sólo se incluyeron niños menores de 5 años y no incorporó participantes de la Orinoquia y la Amazonia colombianas; por tanto, se necesitan estudios en dichas regiones y en un rango de edad más amplio para describir en forma completa la situación del país.

Respecto al parasitismo intestinal en Colombia, los resultados de un estudio, representativo para 
el país, efectuado por el Instituto Nacional de Salud entre 1995 y1996 en niños menores de 5 años mostraron lo siguiente: Giardia lamblia, $21,2 \%$; A. lumbricoides, 3,5\%; Entamoeba histolytica, 3,4\%; T. trichiura, 2,4\% (19). Un estudio efectuado en 2000 con población hospitalaria en el hospital local del municipio de Valle del Guamuez, Putumayo, lugar en donde se realizó el presente estudio, mostró que las frecuencias de algunos parásitos eran más altas que las informadas en el estudio nacional: $T$. trichiura, 9,5\%; A. lumbricoides, 7,2\%; uncinarias 10,1\%; S. stercoralis, 3,6\%; G. lamblia, 14,6\% y E. histolytica, 44,6\% (20). Cabe destacar que el método de diagnóstico parasitológico utilizado en este estudio tiene una baja sensibilidad, pues es un examen directo de una muestra con solución salina sin emplear métodos de concentración ni coloraciones y, por tanto, cabría suponer que la frecuencia debe ser aún mayor y, de acuerdo con lo expuesto anteriormente, también podríamos esperar frecuencias de desnutrición, especialmente desnutrición crónica, relativamente altas respecto a las del país en general.

El presente estudio se desarrolló entre agosto y diciembre de 2001 con niños del municipio Valle del Guamuez, Putumayo. Los objetivos fueron: determinar la frecuencia de parasitismo intestinal, determinar la frecuencia de índices antropométricos bajos y 3) identificar posibles asociaciones entre parasitismo intestinal, nivel socioeconómico y la presencia de índices antropométrico bajos, especialmente de baja talla para la edad.

\section{Materiales y métodos}

\section{Área y sujetos de estudio}

El presente es un estudio descriptivo de corte transversal que se realizó con niños de dos escuelas públicas, Escuela La Parke y Escuela La Libertad, y niños de 2 a 7 años de los barrios aledaños a éstas, ubicadas en el perímetro urbano de la localidad de La Hormiga, municipio Valle del Guamuez, departamento del Putumayo, Colombia. La Hormiga es una población que se encuentra en la Amazonia colombiana; su clima es tropical húmedo, tiene una población aproximada de 9.000 habitantes (de los cuales 5.400, aproximada- mente, son menores de 20 años). Según la oficina local del Sistema de Selección de Beneficiarios para Programas Sociales (Sisben, encuesta socioeconómica utilizada por el gobierno nacional de Colombia, ver descripción posterior), la mayor parte de su población se encuentra clasificada en estratos socioeconómicos bajos. Una descripción más detallada de sus características socioeconómicas, demográficas y geográficas se realizó en un estudio previo (20).

Un total de 354 niños con edades entre 2 y 16 años se inscribieron al estudio, previa explicación verbal de los objetivos y métodos a utilizar y obtención de consentimiento por parte de los padres. La siguiente información fue solicitada a cada participante: nombre, sexo, fecha de nacimiento (obtenida a partir del registro civil de nacimiento que expide la Registraduría Nacional del Estado Civil de Colombia), área de residencia (urbana o rural), puntaje obtenido en la encuesta Sisben (tomado del certificado escrito expedido por la oficina local del Sisben); además, los niños se pesaron y tallaron según la descripción que se hace más adelante. Finalmente, a cada niño se le entregaron cuatro recipientes plásticos con una capacidad aproximada de $180 \mathrm{ml}$, marcados con su nombre y un número de identificación y se les pidió que recogieran cuatro muestras de materia fecal con una cantidad mínima aproximada de 30 g a lo largo de ocho días en fechas preestablecidas.

Todos los niños que presentaron parásitos intestinales recibieron tratamiento.

\section{Antropometría}

Las medidas antropométricas se obtuvieron según los procedimientos estandarizados (21).

El peso se obtuvo en horas de la mañana estando los niños descalzos y en ropa interior; debían estar erectos, con sus manos a los lados y el peso de su cuerpo distribuido en ambos pies. Se utilizó una balanza Foehnle-Vlig Modelo 06falg, con una precisión de $200 \mathrm{~g}$.

La talla se tomó utilizando un tallímetro con pieza cefálica móvil; los niños se encontraban descalzos, en ropa interior, sin accesorios para el cabello, totalmente erectos, con sus manos a 
los lados y el peso de su cuerpo distribuido en ambos pies; debían tener cuatro puntos de contacto sobre la pieza vertical del tallímetro (occipucio, espalda, glúteos y talones). Se midió con una precisión de $5 \mathrm{~mm}$.

La edad se calculó en meses con dos cifras decimales a partir de la fecha de nacimiento y la fecha de medida. Los resultados se presentarán a todo el grupo de participantes, estratificados en tres subgrupos de edad: preescolares, niños y niñas de 24 a 59,99 meses (de 2 a $<5$ años); escolares, niños de 60 a 137,99 meses (de 5 a < 11,5 años), y niñas de 60 a 119,99 meses (de 5 a $<10$ años), y adolescentes, niños mayores de 137,99 meses ( $>=11,5$ años) y niñas mayores de 119,99 meses ( $>=10$ años).

Con esta información se calcularon los siguientes índices antropométricos como puntajes $Z$ respecto de la media de referencia internacional recomendada por la NCHS/OMS (22-24): HAZ (talla para la edad), WAZ (peso para la edad), WHZ (peso para la talla). WHZ se calculó para niños menores de 138 meses (11,5 años) y niñas menores de 120 meses (10 años); la razón para esta limitación es que el peso para la talla no es independiente de la edad en niños mayores y hay gran variabilidad en los pesos y tallas cuando los niños entran en la pubertad (24). HAZ y WAZ se calcularon para todos los niños. Se consideraron bajos aquellos índices que fueran menores de -2 DE o más en relación con la media de referencia, según las recomendaciones de la OMS $(22,24)$.

Con respecto a la terminología utilizada en la presentación de los resultados, queremos hacer algunas consideraciones. Primero, en idioma inglés, la OMS recomienda la utilización de los términos "stunting/stunted", "wasting/wasted" y "underweight" (cuya traducción al español sería "acortamiento/acortado", "adelgazamiento/ adelgazado" y "bajo peso", respectivamente) cuando el valor de los índices HAZ, WHZ y WAZ sea menor al punto de corte de $-2 \mathrm{DE}$ respecto de la media de referencia. Adicionalmente, recomienda evitar el uso de los términos desnutrición crónica, desnutrición aguda y desnutrición global cuando sólo se cuente con el valor de los índices antropométricos, ya que esta presunción representa deducciones que no siempre son correctas. Idealmente, la definición del punto de corte para diferenciar "normalidad nutricional" de "desnutrición" debe basarse en consideraciones biológicas, tales como el incremento en el riesgo de mortalidad o de daño funcional y no solamente en una mera separación por parámetros estadísticos. El uso del punto de corte debe considerarse como una herramienta de tamizaje para identificar aquellos niños que probablemente puedan estar desnutridos $(22,24)$. Por lo expuesto anteriormente, al presentar los resultados utilizamos los términos descriptivos "baja talla para la edad", "bajo peso para la talla" y "bajo peso para la edad".

\section{Análisis parasitológicos}

Las cuatro muestras de materia fecal de cada sujeto fueron observadas bajo el microscopio de luz en examen directo; de cada muestra se prepararon dos láminas con solución salina normal y dos teñidas con lugol parasitológico; la lectura se realizó en las 2 horas siguientes a la entrega de la muestra por parte de los participantes.

Debido a la baja sensibilidad del examen directo para la detección de larvas de S. stercoralis, aproximadamente del $20 \%$ (25), adicionalmente todas las muestras se procesaron por el método de Baermann modificado, que con cuatro muestras tiene una sensibilidad superior al $70 \%$ y es uno de los métodos de diagnóstico recomendado para este parásito $(25,26)$. La técnica utilizada corresponde al método de Baermann modificado, según la descripción hecha por Watson y colaboradores (27) y Kaminsky (25).

Ya que ocasionalmente se pueden encontrar larvas rabditiformes de uncinarias en los especímenes, cuando se dejan por algún tiempo antes de analizar (en este estudio, en algunos casos, la lectura del sedimento en la técnica de Baermann se llevó a cabo incluso hasta 24 horas después de recogida la muestra), se utilizaron características morfológicas distintivas de cada especie para identificarlas: una pequeña cápsula bucal y un gran primordio genital en las larvas de $S$. stercoralis y una gran cápsula bucal y pequeño primordio genital en las larvas de uncinarias (28). 
Blastocystis hominis es un protozoario cuyo papel patogénico en humanos se discute $(29,30)$, por ello en este estudio fue asumido como comensal y no como parásito intestinal.

No se realizó la técnica de la cinta engomada de Graham para el diagnóstico de oxiuros.

\section{Información socioeconómica}

El gobierno colombiano diseñó una encuesta para estimar el nivel socioeconómico de los individuos y familias del país, con el fin de identificar población vulnerable para adjudicar los subsidios estatales. Esta encuesta se conoce como Sisben (Sistema de Identificación de Beneficiarios de los Subsidios del Estado) y consta de 62 preguntas agrupadas en cuatro variables: vivienda, servicios, educación y seguridad social, ingreso y composición familiar. Las variables para la construcción del indicador se determinaron con base en la información obtenida en la Encuesta de Caracterización Socioeconómica aplicada a 25.000 familias de todo el país a mediados de 1993. Mediante técnicas estadísticas se determinaron cuáles de todas estas variables eran las que más influían en el nivel de pobreza da cada una de las familias de Colombia (31).

Los resultados de la encuesta se ingresan a un programa diseñado para tal fin que arroja un puntaje entre 0 y 100 . Entre más cerca se encuentre de 0 mayor será el nivel de pobreza y viceversa; los puntajes obtenidos son finalmente categorizados en seis niveles. Los niveles 1 y 2 corresponden a las familias que se encuentran en extrema pobreza y en situación de pobreza, respectivamente; a estos dos niveles pertenecen las familias que tienen una o más necesidades básicas insatisfechas (NBI) o que disponen de un ingreso familiar suficiente para comprar sólo la canasta básica de alimentos definida como tal por el Departamento Administrativo Nacional de Estadística.

En este estudio utilizamos el puntaje obtenido en la encuesta del Sisben como medida del nivel socioeconómico de los participantes; para los análisis estadísticos este puntaje es asumido como una variable de intervalos.

\section{Análisis estadístico}

La información obtenida se analizó mediante el programa Epi Info 2000 versión 1.1.2 (32). El cálculo de la edad y de los índices antropométricos HAZ, WAZ y WHZ, se efectuó con la calculadora nutricional del programa. La comparación de las medias de los índices antropométricos entre los diferentes grupos se hizo utilizando la prueba t en el caso de dos grupos y el análisis de la varianza (Anova) cuando había tres o más grupos. Las relaciones entre el puntaje en la encuesta del Sisben, el número de parásitos y el valor de los índices antropométricos se evaluaron mediante análisis de regresión simple. La prueba de ji cuadrado se utilizó para comparar proporciones entre grupos.

Cuando se encontraron asociaciones estadísticamente significativas entre los valores de los índices antropométricos y alguna de las variables de estudio, se procedió a realizar análisis estratificado por los niveles de las otras variables que hubieran resultado significativas con el fin de descartar factores de confusión.

\section{Resultados}

Del total inicial, sólo 237 participantes cumplieron los requisitos, 106 fueron excluidos porque no suministraron las cuatro muestras de materia fecal y los once restantes se excluyeron por inconsistencias en la información suministrada (principalmente, por no presentar el registro civil de nacimiento). Las características generales de estos niños se muestran en el cuadro 1. La distribución por sexo fue prácticamente idéntica (50,6\% niños, $49,4 \%$ niñas); por grupo de edad se observó que más de la mitad de los participantes se clasifican como escolares. En 149 de los 237 participantes (62,9\%), se obtuvo el puntaje en la encuesta Sisben, con valores que oscilaron entre 15 y 47 puntos. Teniendo en cuenta el lugar de procedencia (urbana 93,2\%) y el estrecho rango del puntaje en la encuesta Sisben (32 puntos; rango total de la encuesta 100 puntos), podemos considerar a la población estudiada relativamente homogénea desde el punto de vista socioeconómico. Además, encontramos que todos los participantes se encuentran en los niveles más 
Cuadro 1. Características demográficas y socioeconómicas de los participantes en el estudio $n=237$.

\begin{tabular}{lcrrrrr}
\hline Grupos de edad & \multirow{2}{*}{ Frecuencia } & \multicolumn{2}{c}{ Sexo } & & \multicolumn{2}{c}{ Area de Residencia } \\
\cline { 3 - 4 } & & Masculino & Femenino & & Urbana & \multicolumn{1}{c}{ Rural } \\
\hline Preescolares & $26(11 \%)$ & $13(50 \%)$ & $13(50 \%)$ & & $24(92,3 \%)$ & $2(7,7 \%)$ \\
Escolares & $152(64,1 \%)$ & $89(58,6 \%)$ & $63(41,4 \%)$ & & $141(92,8 \%)$ & $11(7,2 \%)$ \\
Adolescentes & $59(24,9 \%)$ & $18(30,5 \%)$ & $41(69,5 \%)$ & & $56(94,9 \%)$ & $3(5,1 \%)$ \\
Total & $237(100 \%)$ & $120(50,6 \%)$ & $117(49,4 \%)$ & & $221(93,2 \%)$ & $16(6,8 \%)$ \\
\hline
\end{tabular}

bajos de la escala (Sisben 1 y Sisben 2), que corresponden a aquellos con condiciones socioeconómicas desfavorables. Los análisis estadísticos que incluyeron el puntaje de la encuesta Sisben sólo utilizaron los 149 sujetos en quienes este valor se obtuvo.

Los resultados de los exámenes parasitológicos se describen en el cuadro 2. Es notoria la alta prevalencia de parásitos intestinales entre la población estudiada; el resultado fue positivo para, por lo menos, un parásito en 204 de los 237 casos, lo que equivale al $86,1 \%$ (en este resultado están excluidos los comensales intestinales). G. lamblia fue el parásito más frecuente, pues se encontró en más de la mitad de los sujetos de estudio $(54,4 \%)$. Es de resaltar que entre los helmintos, aquéllos que se transmiten a través de la piel $(S$. stercoralis $47,3 \%$ y uncinarias $28,7 \%$ ) fueron más frecuentes que aquéllos que se transmiten por vía fecal-oral (tricocéfalos, 13,5\%; Ascaris, 9,7\%, y oxiuros, $4,2 \%$ ). Por grupos de edad, los escolares y adolescentes presentaron la mayor frecuencia de parasitación y los adolescentes la tasa más alta de poliparasitismo intestinal, además de las frecuencias por grupo más altas para uncinarias, tricocéfalos y oxiuros. Los comensales intestinales fueron, incluso, más frecuentes que los parásitos; se encontró uno o más de ellos en 213 de los 237 niños (89,9\%).

En el cuadro 3 se presentan los resultados de la evaluación nutricional. Se encontraron altas frecuencias de índices antropométricos bajos, especialmente de baja talla para la edad y bajo peso para la edad $(29,5$ y $10,1 \%$, respectivamente). Se observó cómo las frecuencias de baja talla para la edad y bajo peso para la edad se incrementaron al aumentar la edad del grupo: mayor en adolescentes que en escolares y en éstos, a su vez, mayor que en preescolares; por ejemplo, para baja talla para la edad, las frecuencias son $45,8,24,3$ y $23,1 \%$, respectivamente. El análisis estadístico mediante el análisis de la varianza (Anova), comparando la media de los índices antropométricos HAZ y WAZ entre los grupos de edad, mostró que esta diferencia era estadísticamente significativa ( $p=0,0027$ para HAZ y $p=0,0009$ para $W A Z$ ), pues las medias de los índices en preescolares fueron mayores que en los otros dos grupos y en escolares, mayores que en adolescentes. La frecuencia de bajo peso para la talla fue comparativamente menor y no hubo diferencias significativas entre los grupos de edad (el índice se calculó para los 178 participantes que se encontraron en los rangos de edad requeridos, 26 preescolares y 152 escolares).

\section{Relaciones entre parasitismo intestinal, nivel socioeconómico y estado nutricional}

Las asociaciones entre parásitos intestinales, nivel socioeconómico (estimado por la encuesta Sisben) y el estado nutricional (estimado a través de los índices HAZ, WAZ, y WHZ) se presentan en el cuadro 4.

Excluyendo la edad, la variable que muestra la mayor correlación con los índices HAZ y WAZ es el número de parásitos $(r=-0,214, p=0,001 ; r=-$ $0,224, p<0,001$, respectivamente), lo cual indica que en la población de estudio la presencia de un mayor número de parásitos se asocia con un menor valor en estos dos índices antropométricos. El cuadro 5 muestra la media y DE de los dos índices respecto al número de parásitos. Esta asociación se presentó en el grupo total y el subgrupo de escolares para los dos índices, así 
Cuadro 2. Resultado (\%) de los exámenes parasitológicos en la población estudiada.

\begin{tabular}{|c|c|c|c|c|}
\hline Parásito ${ }^{1}$ & $\begin{array}{l}\text { Todas las edades } \\
\qquad n=237\end{array}$ & $\begin{array}{c}\text { Preescolares } \\
n=26\end{array}$ & $\begin{array}{c}\text { Escolares } \\
n=152\end{array}$ & $\begin{array}{l}\text { Adolescentes } \\
\quad \mathrm{n}=59\end{array}$ \\
\hline Giardia lamblia & 54,4 & $61,5^{\star *}$ & 53,3 & 54,2 \\
\hline Strongyloides stercoralis ${ }^{2}$ & 47,3 & 30,8 & $49,3^{* *}$ & 49,2 \\
\hline Uncinarias & 28,7 & 23,1 & 27,6 & $33,9^{* *}$ \\
\hline Trichuris trichiura & 13,5 & 3,8 & 11,2 & $23,7^{* *}$ \\
\hline Entamoeba histolytica o dispar & 11,4 & 11,5 & $11,8^{\star *}$ & 10,2 \\
\hline Ascaris lumbricoides & 9,7 & $11,5^{\star *}$ & 9,9 & 8,5 \\
\hline Enterobius vermicularis & 4,2 & 3,8 & 3,3 & $6,8^{* *}$ \\
\hline \multicolumn{5}{|l|}{ Número de parásitos } \\
\hline 0 & 13,9 & 19,2 & 12,5 & 15,3 \\
\hline 1 & 31,6 & 34,6 & 36,2 & 18,6 \\
\hline 2 & 30,8 & 26,9 & 29,6 & 35,6 \\
\hline 3 & 19,4 & 19,2 & 16,4 & 27,1 \\
\hline 4 & 3,4 & - & 4,6 & 1,7 \\
\hline 5 & 0,8 & - & 0,7 & 1,7 \\
\hline Cualquier parásito ${ }^{3}$ & 86,1 & 80,8 & $87,5^{\star *}$ & 84,7 \\
\hline Poliparasitismo intestinal ${ }^{4}$ & 54,4 & 46,2 & 51,3 & $66,1^{\star *}$ \\
\hline Comensales intestinales & & & Todas las edades & \\
\hline Blastocystis hominis & & & 81,9 & \\
\hline Endolimax nana & & & 50,2 & \\
\hline Entamoeba coli & & & 26,6 & \\
\hline
\end{tabular}

1 Resultados obtenidos por examen directo, cuatro muestras por cada paciente.

2 Para su diagnóstico adicionalmente se utilizó el método de Baermann modificado, cuatro muestras por cada paciente.

3 Resultado positivo para cualquier parásito intestinal de los arriba mencionados.

4 Poliparasitismo intestinal, presencia de dos o más parásitos de los arriba mencionados.

** Grupo de edad en que la frecuencia es mayor.

Cuadro 3. Frecuencia (\%) de índices antropométricos bajos en los sujetos de estudio.

\begin{tabular}{lcccc}
\hline & \multicolumn{2}{c}{ Baja talla para la edad } & Bajo peso para la talla & Bajo peso para la edad \\
\cline { 2 - 3 } & Severa ${ }^{1}$ & Total $^{2}$ & & \\
\hline Todas las edades $\mathrm{n}=237$ & 7,2 & 29,5 & $2,5^{3}$ & 10,1 \\
Preescolares $\mathrm{n}=26$ & 3,8 & 23,1 & $3,8^{* *}$ & 3,8 \\
Escolares $\mathrm{n}=152$ & 6,6 & 24,3 & 3,3 & 7,2 \\
Adolescentes $\mathrm{n}=59$ & $10,2^{* *}$ & $45,8^{* *}$ & & $20,3^{* *}$ \\
\hline
\end{tabular}

1 Niños que están 3 DE o más por debajo de la media de referencia.

2 Niños que están 2 DE o más por debajo de la media de referencia; incluye a los niños que están 3 DE o más por debajo de la media de referencia.

3 Porcentaje calculado para 178 niños que cumplen con el requisito arriba mencionado.

** Grupo de edad en que la frecuencia es mayor.

como en el subgrupo de adolescentes para el índice WAZ.

De manera individual, dos parásitos mostraron asociaciones estadísticamente significativas en el análisis preliminar: S. stercoralis, con el índice HAZ para el grupo total y con el índice WAZ para el subgrupo de adolescentes y uncinarias con los índices HAZ y WAZ para el grupo total y el subgrupo de escolares. Sin embargo, al realizar el análisis estratificado por los niveles de la variable presencia/ausencia de poliparasitismo intestinal, sólo la asociación entre uncinarias y el índice WAZ en el subgrupo de escolares y nivel "PPI=presente" continuó siendo significativa $(p=0,015)$.

Respecto al puntaje en la encuesta Sisben, se presentó correlación significativa con el índice HAZ para el grupo total $(r=0,173 p=0,027)$; es decir, a 
Cuadro 4. Correlaciones entre parasitismo intestinal, nivel socioeconómico y estado nutricional.

\begin{tabular}{|c|c|c|c|c|}
\hline Variable & Prueba estadística & $\begin{array}{c}\mathrm{HAZ} \\
\boldsymbol{p}^{\star}\end{array}$ & $\begin{array}{c}\text { WAZ } \\
\boldsymbol{p}^{\star}\end{array}$ & $\begin{array}{c}\text { WHZ } \\
\boldsymbol{p}^{*}\end{array}$ \\
\hline \multicolumn{5}{|l|}{ Giardia } \\
\hline Todas las edades & Prueba $t$ & 0,422 & 0,352 & 0,642 \\
\hline Preescolares & Prueba $t$ & 0,419 & 0,506 & 0,996 \\
\hline Escolares & Prueba $t$ & 0,133 & 0,193 & 0,679 \\
\hline Adolescentes ${ }^{1}$ & Pruebat & 0,977 & 0,841 & 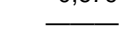 \\
\hline \multicolumn{5}{|l|}{ S. stercoralis } \\
\hline Todas las edades & Prueba $t$ & 0,036 & 0,082 & 0,468 \\
\hline Preescolares & Pruebat & 0,877 & 0,642 & 0,793 \\
\hline Escolares & Pruebat & 0,137 & 0,544 & 0,496 \\
\hline Adolescentes & Pruebat & 0,182 & 0,035 & - \\
\hline \multicolumn{5}{|l|}{ Uncinarias } \\
\hline Todas las edades & Prueba $t$ & 0,012 & 0,016 & 0,227 \\
\hline Preescolares & Prueba $t$ & 0,634 & 0,828 & 0,524 \\
\hline Escolares & Pruebat & 0,028 & 0,005 & 0,147 \\
\hline Adolescentes & Prueba t & 0,429 & 0,949 & 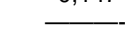 \\
\hline \multicolumn{5}{|l|}{ Tricocéfalos } \\
\hline Todas las edades & Pruebat & 0,092 & 0,053 & 0,685 \\
\hline Preescolares & Prueba $t$ & & & \\
\hline Escolares & Pruebat & 0,211 & 0,355 & 0,905 \\
\hline Adolescentes & Pruebat & 0,854 & 0,231 & \\
\hline \multicolumn{5}{|l|}{ E. histolytica } \\
\hline Todas las edades & Prueba $t$ & 0,139 & 0,179 & 0,573 \\
\hline Preescolares & Pruebat & 0,483 & 0,525 & 0,169 \\
\hline Escolares & Pruebat & 0,149 & 0,225 & 0,835 \\
\hline Adolescentes & Pruebat & 0,728 & 0,180 & 0,00 \\
\hline \multicolumn{5}{|c|}{ Ascaris lumbricoides } \\
\hline Todas las edades & Prueba t & 0,446 & 0,302 & 0,115 \\
\hline Preescolares & Prueba t & 0,069 & 0,187 & 0,958 \\
\hline Escolares & Pruebat & 0,832 & 0,244 & 0,106 \\
\hline Adolescentes & Pruebat & 0,694 & 0,198 & 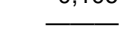 \\
\hline \multicolumn{5}{|l|}{ Oxiuros } \\
\hline Todas las edades & Prueba $t$ & 0,228 & 0,938 & 0,810 \\
\hline Edad & Regresión simple & $r=-0,283$ & $r=-0,224$ & $r=0,223$ \\
\hline & & $p=0,0001$ & $p=0,0004$ & $p=0,002$ \\
\hline \multicolumn{5}{|c|}{ Número de parásitos } \\
\hline \multirow[t]{2}{*}{ Todas las edades } & Regresión simple & $r=-0,214$ & $r=-0,224$ & $r=-0,051$ \\
\hline & & $p=0,001$ & $p<0,001$ & $p=0,563$ \\
\hline \multirow[t]{2}{*}{ Preescolares } & Regresión simple & $r=0,073$ & $r=0,155$ & $r=0,144$ \\
\hline & & $p=0,763$ & $p=0,333$ & $p=0,403$ \\
\hline \multirow[t]{2}{*}{ Escolares } & Regresión simple & $r=0,245$ & $r=0,221$ & $r=0,088$ \\
\hline & & $p=0,002$ & $p=0,003$ & $p=0,377$ \\
\hline \multirow[t]{2}{*}{ Adolescentes } & Regresión simple & $r=-0,167$ & $r=-0,224$ & 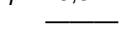 \\
\hline & & $p=0,211$ & $p=0,036$ & \\
\hline \multicolumn{5}{|l|}{ SISBEN ${ }^{2}$} \\
\hline \multirow[t]{2}{*}{ Todas las edades } & Regresión simple & $r=0,171$ & $r=0,140$ & $r=-0,002$ \\
\hline & & $p=0,027$ & $p=0,109$ & $p=0,897$ \\
\hline \multirow[t]{2}{*}{ Preescolares } & Regresión simple & $r=0,141$ & $r=-0,170$ & $r=-0,412$ \\
\hline & & $p=0,601$ & $p=0,540$ & $p=0,127$ \\
\hline \multirow[t]{2}{*}{ Escolares } & Regresión simple & $r=0,173$ & $r=0,141$ & $r=0,004$ \\
\hline & & $p=0,111$ & $p=0,161$ & $p=0,821$ \\
\hline \multirow[t]{2}{*}{ Adolescentes } & Regresión simple & $r=0,282$ & $r=0,245$ & $\longrightarrow$ \\
\hline & & $p=0,078$ & $p=0,120$ & \\
\hline \multicolumn{5}{|l|}{ Procedencia } \\
\hline Todas las edades & Prueba $t$ & 0,132 & 0,505 & 0,448 \\
\hline
\end{tabular}

* Valor $p$ correspondiente a la comparación de las medias para los diferentes índices antropométricos obtenidos en las poblaciones de parasitados y no parasitados, además se presenta el coeficiente de correlación simple cuando se empleó la regresión simple.

1 Para este grupo no se calculo el índice WHZ por estar fuera del rango de edad.

2 La correlación entre el puntaje en la encuesta SISBEN y los índices HAZ y WAZ se hizo con los sujetos con esta información disponible: 149 para el grupo total, 15 preescolares, 93 escolares y 41 adolescentes. Los cálculos para el índice WHZ se hicieron en quienes, además de tener la información del SISBEN disponible, estuvieran dentro del rango de edad: 108 para el grupo total, 15 para preescolares y 93 para escolares. 
Cuadro 5. Media y desviación estándar de los índices HAZ y WAZ respecto al número de parásitos en el grupo total ${ }^{*}$.

\begin{tabular}{cccc}
\hline $\begin{array}{l}\text { Número de } \\
\text { parásitos }\end{array}$ & $\mathbf{n}$ & HAZ & WAZ \\
& & $\mathbf{X} \pm \mathbf{2} \mathbf{~ D E}$ & $\mathbf{X} \pm \mathbf{2}$ DE \\
\hline 0 & 33 & $-0,997 \pm 2,492$ & $-0,525 \pm 1,972$ \\
1 & 75 & $-1,232 \pm 2,554$ & $-0,644 \pm 2,220$ \\
2 & 73 & $-1,467 \pm 1,796$ & $-0,809 \pm 1,728$ \\
3 & 46 & $-1,732 \pm 2,188$ & $-1,084 \pm 1,604$ \\
4 y $5^{1}$ & 10 & $-1,674 \pm 2,174$ & $-1,487 \pm 1,623$ \\
\hline
\end{tabular}

* Estadísticas calculadas para los 237 participantes.

${ }^{1}$ Los niños con 4 y 5 parásitos se agruparon, ya que sólo dos de ellos presentaban cinco parásitos.

mayor puntaje en la encuesta, mayor el valor del índice HAZ.

La procedencia de los participantes no se relacionó significativamente con el valor en ninguno de los tres índices antropométricos.

Para el índice WHZ sólo se encontró una asociación positiva estadísticamente significativa con la variable edad: los niños de mayor edad tienden a presentar valores mayores para este índice.

Al realizar el análisis de regresión múltiple para el índice HAZ utilizando la edad, el número de parásitos y el puntaje Sisben como variables predictoras, encontramos que la variabilidad del índice que puede ser explicada por las dos primeras variables tomadas simultáneamente es mayor que la que puede explicar cada una de ellas por separado $\left(R=0,332 ; R^{2}=0,110 ; p=0,008\right.$ al agregar la segunda variable); sin embargo, al adicionar el puntaje Sisben al modelo no se produjo un aumento estadísticamente significativo en la variabilidad explicada $\left(R=0,346 ; R^{2}=0,119\right.$; $\mathrm{p}=0,073$, al agregar la tercera variable). Este último hecho se debe a que la variabilidad del índice explicada por la variable Sisben se solapa con la explicada por la variable número de parásitos, punto que se aprecia mejor al observar el coeficiente de correlación simple entre las variables número de parásitos y puntaje Sisben $(r=-0,173 ; p=0,028)$; es decir, aquéllos con puntajes bajos en la encuesta socioeconómica presentan, en general, un mayor número de parásitos.

Con respecto a la predicción del índice WAZ encontramos una fuerte relación con los índices
HAZ y WHZ ( $r=0,640, p<0,0000001$, y $r=0,574$, $p<0,0000001$, respectivamente). La variabilidad de WAZ, que se explica por los otros dos índices tomados simultáneamente, fue aproximadamente del 95\% ( $R=0,975, R^{2}=0,950 ; p<0,0000001$, al agregar la segunda variable).

\section{Discusión}

Los datos obtenidos en la población de estudio apoyan la relación previamente descrita entre parasitismo intestinal y desnutrición. Sin embargo, como en cualquier otro estudio descriptivo, si bien las asociaciones que encontramos no implican una relación causal sí ayudan a orientar posteriores estudios, así como el manejo tanto individual como colectivo del problema.

A pesar de que encontramos variables independientes relacionadas de una forma estadísticamente significativa con el índice antropométrico HAZ (edad, número de parásitos, puntaje Sisben), el modelo de regresión múltiple utilizado para su predicción sólo alcanza a explicar el $11 \%\left(R^{2}=0,1102\right)$ de la variabilidad del índice. Consideramos que probablemente ello se debe a que, como mencionamos anteriormente, la talla, el peso y el estado nutricional de un individuo en un momento dado dependen de una compleja interacción de variables; además, en el estudio no se incluyeron variables consideradas como predictores importantes, como son el peso al nacer y la talla materna $(1,8,12,15)$.

La fuerte correlación de los índices HAZ y WHZ con el índice WAZ no sorprende si recordamos que este último básicamente está compuesto por los dos primeros y que en este estudio la mayor parte de la variabilidad de WAZ se explica por los otros dos índices $\left(R^{2}=0,950\right)$, similar a lo descrito anteriormente $(22,24)$. Al observar los coeficientes de correlación simple encontramos que tanto los procesos que conducen a falta en la ganancia o pérdida de peso, como los que conducen a falta de crecimiento en estatura, con una ligera preponderancia de este último, fueron los responsables del mal estado nutricional entre la población de estudio.

Nos llama la atención la disminución en el valor de los índices antropométricos HAZ y WAZ, así 
como el incremento en la frecuencia de valores por debajo de -2 DE de la media de referencia internacional, que ocurren con la edad. Este hecho podría reflejar el continuo efecto negativo que sobre el estado nutricional individual tienen múltiples factores nocivos presentes en una población, con lo cual no sólo los niños que están desnutridos no se recuperan, sino que también se produce un deterioro progresivo en el estado nutricional de los niños que todavía no lo están. Este fenómeno se aprecia mejor en un estudio longitudinal realizado con niños en Zambia (Hautvast, 2000), entre quienes la media de los índices antropométricos disminuyó progresivamente a través de tres periodos de observación. Sin duda alguna, éste es un punto importante a tener en cuenta al momento de diseñar estrategias de salud pública tendientes a mejorar el estado nutricional en estas comunidades.

Aunque no encontramos asociaciones estadísticamente significativas entre parásitos intestinales específicos y el estado nutricional de los participantes (con la excepción de la asociación entre uncinarias y el valor del índice WAZ cuando, además, el subgrupo de escolares se encontraba poliparasitado), sí se observó una clara relación entre poliparasitismo intestinal y un menor valor en los índices antropométricos HAZ y WAZ, el cual podría representar el efecto negativo sinérgico de los múltiples parásitos intestinales a lo largo del tiempo, hecho congruente con el estado actual del conocimiento sobre el tema.

El parámetro utilizado en este estudio para valorar el estado socioeconómico de los participantes, el puntaje en la encuesta Sisben, presentó asociación significativa con el valor del índice HAZ (positiva) y con el número de parásitos (negativa); es decir, los individuos con bajos puntajes en la encuesta, bajo nivel socioeconómico, presentan, en general, un mayor número de parásitos y, a su vez, menor valor en el índice antropométrico. Si bien estas relaciones fueron estadísticamente significativas, el valor del coeficiente de correlación fue más bajo de lo que se esperaría ( $r=0,171$ para HAZ-Sisben y $r=-0,173$ para número de parásitosSisben); esto puede deberse a que el grupo en general es relativamente homogéneo en este aspecto. La forma en que el estado socioeconómico de un individuo afecta su estado nutricional incluye una variedad de mecanismos entre los cuales pueden estar: déficit en la calidad y cantidad de alimentos, conductas alimentarias específicas, disponibilidad de servicios sanitarios y de salud y otros, y podría también ejercer algún efecto a través de la presencia o ausencia de parásitos intestinales. Sin duda alguna, la validez de estas asociaciones tiene su principal obstáculo en la utilización de la encuesta misma, ya que ésta se creó con un objetivo diferente; la validez para su uso en estudios de investigación en medicina no ha sido evaluada y no conocemos estudios previos en los que se haya utilizado; además, su efectividad depende de la correcta aplicación de la encuesta por parte de los técnicos encargados. A pesar de estas consideraciones la encontramos útil, ya que es práctica, objetiva y si bien sus resultados no se pueden utilizar para hacer comparaciones en el ámbito internacional, sí se podrían hacer en lo nacional.

Al contrastar los actuales resultados con los de un estudio reciente en la región (20), encontramos una alta frecuencia de parasitismo y poliparasitismo intestinal; sin duda, la mayor sensibilidad de los métodos diagnósticos empleados puede explicar este hecho. Un aspecto llamativo es que los helmintos que se transmiten a través de la piel son más frecuentes que los que se transmiten por vía fecal-oral; la costumbre difundida en la región de no utilizar calzado en forma permanente puede en parte explicar este hallazgo. Al comparar nuestros resultados con los obtenidos en el estudio del Instituto Nacional de Salud de 1995-1996 (19), en el grupo de preescolares, encontramos que las frecuencias de giardias, áscaris, tricocéfalos y E. histolytica son superiores en la región de estudio que las informadas en el país. Para tener una idea de la situación actual del parasitismo intestinal en las regiones, revisamos los resultados de algunos estudios llevados a cabo en países sudamericanos. En Paraguay (Canese, 1999), con 3.744 niños entre los 4 y 15 años se informó: G. lamblia, 24,6\%; uncinarias 9,5\%; Ascaris, 7,7\%; y tricocéfalos $0,8 \%$ (33). En Venezuela, un estudio efectuado para determinar la prevalencia de $G$. lamblia (Devera, 1998) encontró este parásito en 
$10,5 \%$ de 493 niños con edades entre 5 a 14 años (34). En Argentina, Borda y colaboradores informan los resultados de 207 niños con edades entre los 3 y 16 años: G. lamblia, 29\%; uncinarias, $27 \%$ y Ascaris, 0,5\% (35). Finalmente, en Chile, en 1994, con 244 niños entre los 6 y 14 años se encontraron las siguientes frecuencias: tricocéfalos, 32\%; $G$. lamblia, 27,9\%, y Ascaris, $12,7 \%$ (36). La sensibilidad de los métodos parasitológicos de los estudios venezolano y argentino es comparable a la nuestra (con la excepción de la valoración de S. stercoralis); la sensibilidad de los métodos usados en los estudios paraguayo y chileno es menor. Observamos cómo, en general, las frecuencias que informamos son superiores a las encontradas en estos países, con la excepción de las frecuencias de tricocéfalos y áscaris que se informan en el estudio chileno.

Respecto a la frecuencia de índices antropométricos bajos, el panorama es similar; encontramos en la población de estudio frecuencias mayores que las estimadas para Suramérica en 2000 y que las informadas por la ENDS 2000 para Colombia; llama la atención cómo la frecuencia de baja talla para la edad en esta población es similar a la informada para el país por el Ministerio de Salud durante el periodo 19651966 (17). Al comparar los resultados de la ENDS 2000 con las del subgrupo de preescolares de nuestro estudio, mediante la prueba de ji cuadrado, encontramos que estas diferencias no son estadísticamente significativas $(p>0,05)$. Sin embargo, si tomamos las frecuencias del grupo total de este estudio, las diferencias son estadísticamente significativas tanto para baja talla para la edad como para bajo peso para la talla y bajo peso para la edad $(p<0,05)$.

En conclusión, encontramos que entre la población de estudio las frecuencias de parasitismo intestinal y bajos índices antropométricos, especialmente baja talla para la edad y bajo peso para la edad, son mayores que las informadas para nuestro país en fecha reciente por otros autores. Tanto el poliparasitismo intestinal como el bajo nivel socioeconómico pueden ser dos importantes factores que contribuyen al mal estado nutricional de estos niños y, quizá, lo sean para los que habitan regiones con características similares. Además, como ya se ha encontrado en otros estudios, se observa cómo las frecuencias de baja talla para la edad y bajo peso para la edad aumentan con la edad de los participantes, hecho que puede representar la persistencia en el tiempo de factores nocivos para el estado nutricional entre la población estudiada. Nuevos estudios, especialmente con diseños longitudinales, pueden contribuir a dilucidar varios interrogantes como son la secuencia temporal entre parasitismo intestinal y desnutrición, la importancia relativa del parasitismo intestinal en el estado nutricional de quienes los presentan y, finalmente, qué otros factores contribuyen a un mal estado nutricional y su presentación a lo largo de la vida de un individuo. Llamamos especialmente la atención hacia la parasitación por S. stercoralis, una infección relativamente silenciosa y abandonada tanto en el campo investigativo como en programas de control, ya que nuestros resultados sugieren que, al menos en algunas regiones del país, puede ser un problema de salud pública mayor del que creemos.

\section{Agradecimientos}

Agradecemos a los participantes, padres de familia, rectores de las escuelas y líderes comunitarios por su colaboración en el desarrollo de este trabajo. Agradecemos la colaboración del director del Hospital Sagrado Corazón de Jesús en La Hormiga, Putumayo, Dr. José Víctor Pérez, por facilitarnos las instalaciones necesarias para el procesamiento de las muestras y la ayuda en la consecución de los antiparasitarios. A la oficina local de la UNICEF, La Hormiga, por colaborar en la coordinación y ejecución del proyecto. A las enfermeras jefe Marcelina Cuarán Inagán y Arabelly Montoya, quienes participaron activamente en el trabajo de campo.

\section{Referencias}

1. Stephenson LS, Latham MC, Ottesen EA. Malnutrition and parasitic helminth infections. Parasitology 2000; 121:23-38.

2. Walker SP, Robinson RD, Powell CA, GranthamMcGregor SM. Stunting, intestinal parasitism and the home environment. Trans R Soc Trop Med Hyg 1992; $86: 331-2$.

3. Stephenson LS, Latham MC, Kurz KM, Kinoti SN, Brigham H. Treatment with a single dose of albendazole 
improves growth in Kenyan schoolchildren with hookworms, Trichuris trichiura, and Ascaris lumbricoides infections. Am J Trop Med Hyg 1989;41:78-87.

4. Stephenson LS, Latham MC, Kurz KM, Kinoti SN, Brigham H. Improvements in physical fitness of Kenyan schoolboys infected with hookworms, Trichuris trichiura, and Ascaris lumbricoides following a single dose of albendazole. Trans R Soc Trop Med Hyg 1990;84:27782.

5. Northrop-Clewes C, Roosham EK, Mascie-Taylor N, Lunn PG. Antihelmintic treatment of rural Bangladeshi children: effect on host physiology, growth, and biochemical status. Am J Clin Nutr 2001;73:53-60.

6. Stettler N, Schutz Y, Jequier E. Effect of low-level pathogenic helminth infection on energy metabolism in Gambian children. Am J Trop Med Hyg 1998;58:476-9.

7. Coop RL, Holmes PH. Nutrition and parasite interaction. Int J Parasitol 1996;26:951-62.

8. Hautvast JLA, Tolboom JJM, Kafwembe EM, Musonda RM, Mwanakasale V, Staveren WAV, et al. Severe linear growth retardation in rural Zambian children: the influence of biological variables. Am J Clin Nutr 2000; 71:550-9.

9. Gendrel D, Richard-Lenoble D, Kombila M, Dupont C, Moreno JL, Gendrel C, et al. Influence of intestinal parasites on lactose absorption in well-nourished African children. Am J Trop Med Hyg 1992;46:137-40.

10. Solomons NW. Pathways to the impairment of human nutritional status by gastrointestinal pathogens. Parasitology 1993;107:19-35.

11. Hlaing T. Ascariasis and childhood malnutrition. Parasitology 1993;107:125-36.

12. Hammond KA. Valoración alimentaria y clínica. In: Mahan LK, Escott-Stump S, editores. Nutrición y Dietoterapia de Krause. Décima edición. México: McGraw-Hill Interamericana; 2001.

13. Oberhelman RA, Guerrero ES, Fernandez ML, Silio $\mathrm{M}$, Mercado D, Komiskey N, et al. Correlations between intestinal parasitosis, physical growth, and psychomotor development among infants and children from rural Nicaragua. Am J Trop Med Hyg 1998;58:470-5.

14. Nokes C, Cooper ES, Robinson BA, Bundy AP. Geohelminthic infection and academic assessment in Jamaican children. Trans R Soc Trop Med Hyg 1991;85:272-3.

15. Stephenson LS, Latham MC, Ottesen EA. Global malnutrition. Parasitology 2000;121:5-22.

16. Crompton DWT. How much human helminthiasis is there in the world? J Parasitol 1999;85:397-403.

17. Mora JO, de Paredes B, de Navarro L, Rodriguez E. Mejora del estado nutricional de los niños colombianos entre 1965 y 1989. Bol Of Sanit Panam 1992;113:197210.
18. Profamilia. Encuesta Nacional de Demografía y Salud 2000. Bogotá: Profamilia; 2000.

19. Castro L, Nicholls S. Deficiencia de hierro, vitamina A y prevalencia de parasitismo intestinal en la población infantil y anemia nutricional en mujeres en edad fértil, Colombia 1995-1996. Santa Fe de Bogotá, D.C: Instituto Nacional de Salud; 1998.

20. Ordóñez LE, Angulo ES. Parasitismo intestinal en Valle del Guamuez y San Miguel, Putumayo, Colombia. Medicina y Laboratorio 2000;9:565-74.

21. Centers for Disease Control. Body Mass Index: How to measure weight, height. 11 pantallas. Disponible en URL: http://www.cdc.gov/nccdphp/dnpa/bmi/measheight.htm, http://www.cdc.gov/nccdphp/dnpa/bmi/ meas-weight.htm.

22. WHO Working Group. Use and interpretation of anthropometric indicators of nutritional status. Bull WHO 1986;64:929-41.

23. Dibley MJ, Goldsby JB, Staehling NW, Trowbridge FL. Development of normalized curves for the international growth reference: historical and technical considerations. Am J Clin Nutr 1987;46:736-48.

24. Gorstein J, Sullivan K, Yip R, De Onis M, Trowbridge P, Fajans $\mathbf{P}$, et al. Issues in the assessment of nutrition status using anthropometry. Bull WHO 1994;72:273-83.

25. Kaminsky RG. Evaluation of three methods for laboratory diagnosis of Strongyloides stercoralis infection. J Parasitol 1993;79:277-80.

26. Dreyer G, Fernandes-Silva E, Alves S, Rocha A, Albuquerque $\mathbf{R}$, Addiss $\mathbf{D}$. Patterns of detection of Strongyloides stercoralis in stool specimens: implications for diagnosis and clinical trials. J Clin Microbiol 1996;34: 2569-71.

27. Watson JM, Al-Hafidh R. A modification of the Baermann funnel technique, and its use in establishing the infection potential of human hookworms carriers. Ann Trop Med Parasitol 1957;41:15-6.

28. Organización Panamericana de la Salud. Parasitología. En: Manual de técnicas básicas para un laboratorio de salud. Publicación científica No 439. Washington, D.C.: Organización Panamericana de la Salud; 1983. p.169.

29. Markell EK, Udkow MP. Blastocystis hominis: pathogen or fellow traveller? Am J Trop Med Hyg 1986;35:102326.

30. Botero D, Restrepo M. Otros protozoos intestinales. En: Parasitosis humanas. Segunda edición. Medellín: Corporación para investigaciones biológicas CIB; 1995.

31. Departamento Nacional de Planeación, Unidad de Desarrollo Social, Misión Social. Sistema de Selección de Beneficiarios para Programas sociales. 111 pantallas. Disponible en: URL: http://www.dnp.gov.co/01_CONT/ INDICADO/MISION.HTM\#5 
32. Dean AG, Arner TG. Epi Info 2000, version 1.1.2: a database and statistics program for public health professionals. Atlanta: Centers for Disease Control and Prevention; 2001.

33. Canese A, Barrios E, Castro L, Canese J. Prevalencia de parásitos intestinales encontrados en niños en Paraguay. Rev Par Microbiol 1999;19:75-81.

34. Devera R, Niebla-Punos G, Nastasi-Vatanese JA, Velásquez-Alvarez VJ, Gonsález-Meneses R. Giar- diasis en escolares de Ciudad Bolívar, estado de Bolívar, Venezuela. Rev Biomed 1998;9:145-50.

35. Borda C, Rea M, Rosa J, Maidana C. Parasitismo intestinal en San Cayetano, Corrientes, Argentina. Bol Oficina Sanitaria Panam 1996;120:110-6.

36. Navarrete N, Torres P. Prevalencia de infección por protozoos y helmintos intestinales en un sector costero de la provincia de Valdivia, Chile. Bol Chil Parasitol 1994; 49:79-80. 